\title{
Use of new scratch test and tensile test for evaluation of bond strength of self- adhesive flowable resin composite for repair of artificial tooth erosion
}

\author{
Yuki MURASE ${ }^{1}$, Hirotomo KOTAKE${ }^{1}$, Shusuke KUSAKABE ${ }^{1}$, Katsushi OKUYAMA², Yukimichi TAMAKI² \\ and Masato HOTTA ${ }^{1}$ \\ ${ }^{1}$ Division of Oral Functional Science and Rehabilitation, Department of Operative Dentistry, Asahi University School of Dentistry \\ ${ }^{2}$ Division of Oral Functional Science and Rehabilitation, Department of Dental Materials Science, Asahi University School of Dentistry \\ Corresponding author, Katsushi OKUYAMA; E-mail: katsu@dent.asahi-u.ac.jp
}

\begin{abstract}
The purpose of this study was to use a new scratch test and tensile test to evaluate the bond strength between artificial erosive enamel or dentin and self-adhesive resin composites as a coating material. Coronal enamel or dentin surface was exposed to an erosive cycle (artificial saliva [AS], pH:7.0 for $6.5 \mathrm{~h}$ and acidic carbonated beverages for 5 min, alternated 3 times per day) for the eroded-surface or stored in AS for the remineralized-surface. Two self-adhesive flowable resin composites, Fusio and LLB-CR6 (prototype), and a conventional flowable resin composite, BEAUTIFIL FLOW with self-etching primer system, Clearfil Mega Bond, were applied to enamel or dentin surfaces; and then the bond strengths were measured. For the eroded-surface, there were no significant differences in bonding strength among all materials, as assessed by the new scratch test. Thus, these self-adhesive flowable resin composites might be useful for coating materials on acid-eroded tooth surfaces.
\end{abstract}

Keywords: Self-adhesive resin composite, Artificial tooth erosion, Scratch test, Tensile bond strength

\section{INTRODUCTION}

Tooth wear is one of the problems in dental practice; and it is on the increase in dental patients, thus the need for treatment. Tooth wear has been divided into several types: abrasion (produced by interaction between the teeth and other substances), attrition (produced during tooth-to-tooth contact), and erosion (produced by a chemical process) ${ }^{1,2)}$. Most tooth wear is due to acid erosion, in which the tooth structure is demineralized by acid due to contact with acidic solutions ${ }^{1}$. There are two factors for acid-mediated erosion: one is extrinsic, e.g., the intake acidic food and beverage; and other, endogenous factor, e.g., vomiting or acid reflux ${ }^{3)}$. As to the acideroded tooth, the loss of enamel and demineralization beneath the superficial surface occur at the same time, thus resulting in destruction of the enamel ${ }^{4)}$.

When acid-based erosion is detected, the intake of acidic food and beverage should be limited to remove the cause; and fluoride for remineralization and increased acid-resistance of the enamel or dentin should be applied $^{5,6)}$. However, if part of the tooth structure has been destroyed by such erosion, this area must be restored by application of a resin composite.

Recently, a novel self-adhesive flowable resin composite including adhesive monomer was developed for bonding with enamel or dentin ${ }^{7,8}$. The eroded tooth is received damage by acid (low $\mathrm{pH}$ ), however there is no caries-affected dentin. Some cases of treatment for an eroded tooth require only minimal intervention by use of an adhesive technique for bonding to the enamel or dentin. Some type of material is coated onto the eroded surface, without the need for a dental filling. There has been no research on the optimal adhesive for eroded enamel or dentin.
Most reports on the bonding between enamel or dentin and resin composites showed the results obtained by use of tests for tensile bond strength or shear bond strength. Kusakabe et al. ${ }^{9,10)}$ reported a new scratch test for assessing the bonding strength of dentin adhesives. In this scratch test (thin-film bond strength test), an indenter is drawn across the coated surface under incremental and progressive loading to generate a scratch. This test might be suitable for assessing the bonding of a thin layer of coating material to a tooth, because sometimes eroded teeth are treated by coating with such a thin layer of material.

The purpose of the present study was to evaluate, by use of this new scratch test and tensile test, the bonding strength between self-adhesive resin composites as a coating material and artificially eroded enamel or dentin as a simulated system of the oral environment.

\section{MATERIALS AND METHODS}

\section{Artificially eroded samples}

The lingual surface of human extracted anterior teeth was grounded with \#600 SiC paper, or coronal human extracted molars were cut perpendicular to the long axis by a low-speed cutting machine (Isomet, Bueler, Lake Bluff, IL, USA) and grounded with \#600 SiC paper to expose the flat enamel or dentin surface (group $\mathrm{E}$ or $\mathrm{D}$, respectively). The study for using human extracted teeth was approved by the Research Ethics Committee of Asahi University School of Dentistry (Approval Number: 245158). The artificially eroded enamel or dentin samples (Ee or De, respectively) were prepared by applying a demineralization cycle using artificial saliva $^{11)}$, which contained $1.5 \mathrm{mM} \mathrm{CaCl}_{2}, 0.9 \mathrm{mM} \mathrm{KH}_{2} \mathrm{PO}_{4}$, $130 \mathrm{mM} \mathrm{KCl}, 20 \mathrm{mM}$ HEPES buffer solution (2-[4-(2- 
hydroxyethyl)-1-piperazinyl] ethane sulfonic acid) and adjusted with $\mathrm{KOH}$ to a $\mathrm{pH}$ of 7.0 and a strongly acidic (pH 2.2) carbonate drink (Coca Cola ${ }^{\circledR}$ : Cola ; Japan Coca Cola, Tokyo, Japan). These $\mathrm{pH}$ values were adjusted or measured by using a combination $\mathrm{pH}$ electrode (Orion 9102BNWP, Thermo Fisher Scientific, Beverly, MA, USA) connected to an expandable ion-analyzer (Orion benchtop multiparameter meter model 350, Thermo Fisher Scientific). The 3-cycle erosion procedure was carried out after immersion in the artificial saliva for 2 $\mathrm{h}$ at $37^{\circ} \mathrm{C}$. Each cycle involved immersion in Coca Cola for $5 \mathrm{~min}$ at $4^{\circ} \mathrm{C}$, a 5 -min rinse with distilled deionized water at room temperature, and storage in the artificial saliva at $37^{\circ} \mathrm{C}$ for $6 \mathrm{~h} 30 \mathrm{~min}$. During storage the sample in the artificial saliva was shaken at $1 \mathrm{~Hz}$ in an incubation shaker (BR-30LF, TAITEC, Koshigaya, Japan). The group $\mathrm{E}$ and $\mathrm{D}$ samples were stored in artificial saliva for same period as the eroded sample to create remineralized enamel (Er) or dentin (Dr) samples. The surface of these before-treated, eroded and remineralized teeth were observed by scanning electron microscopy (SEM; S-4500, HITACHI, Tokyo, Japan) at $\times 500$ or $\times 2,500$ magnification.

\section{Materials used in this study}

Three flowable resin composites were used as a coating material in this study. Two self-adhesive flowable resin composites, i.e., Fusio Liquid Dentin (Fusio; Pentron Clinical, Orange, CA, USA) and a prototype flowable resin composite (LLB-CR6) with primer on Primfil (LLB; Tokuyama Dental, Tokyo, Japan), and a conventional flowable resin composite, BEAUTIFIL FLOW F02 (BF; Shofu, Kyoto, Japan), with Clearfil Mega Bond (MB; Kuraray Noritake, Tokyo, Japan) as a self-etching primer system. The composition of each material is shown in Table 1.

Micro hardness test

For enamel or dentin samples, group E, D, Ee or
De samples were dried just before testing and then examined. For resin samples (Fusio, LLB, and BF), a silicon mold (3.0 $\mathrm{mm}$ diameter, $1.0 \mathrm{~mm}$ height) was filled with a given resin, which was then light cured by use of a light-curing unit (Dentcraft Bluelex, Yoshida, Tokyo, Japan) for $10 \mathrm{~s}$. After curing, the surface was grounded with \#600 SiC paper. The Vickers hardness test was performed for each specimen by using a micro Vickers hardness machine (HM-211, Mitsutoyo, Kawasaki, Japan) with Vickers indenter loaded at $200 \mathrm{mgf}$ for 15 s. For every specimen, 6 points were measured and their average were calculated. Five specimens were evaluated for each enamel/dentin treatment or resin material.

\section{Scratch test for bond strength}

Ten specimens of each tooth sample (Ee, De, Er, Dr) were used for the scratch test. For Fusio samples, a material was applied on the tooth surface, which was mildly airdried, pressed with a clear acrylic plate at 200 gf for 30 $\mathrm{s}$, and then light-cured for $20 \mathrm{~s}$. For LLB samples, the primer was applied to the tooth surface and then dried with mild air immediately. LLB paste was then coated onto the primer-treated surface and pressed and cured in the same manner as for the Fusio sample. For MB with $\mathrm{BF}(\mathrm{MB}+\mathrm{BF})$ samples, a primer of $\mathrm{MB}$ was applied to the tooth surface; and $20 \mathrm{~s}$ later, the surface was mildly airdried. A bonding agent was then applied as a thin layer on the primed-surface and thereafter light-cured for 10 s. Then BF material was coated onto the treated surface, which material was subsequently pressed and cured in the same manner as for the Fusio and LLB samples.

The scratch test for bond strength was performed on each sample according to previous reports ${ }^{9,10)}$. Briefly, a diamond indenter was slid along each test specimen at a sliding speed of $5.25 \mathrm{~mm} / \mathrm{min}$ with loads of $0-30 \mathrm{~N}$. This sliding was performed for a length of approximately $7 \mathrm{~mm}$ by using a thin-film bond strength measurement system (Auto scratch testing machine, CSEM Instruments, Peseux, Switzerland, Fig. 1). The bond strength (thin-

Table 1 Composition of materials used in this study

\begin{tabular}{lll}
\hline \multicolumn{1}{c}{ Material } & \multicolumn{1}{c}{ Composition } & Code \\
\hline \multirow{2}{*}{ Fusio Liquid Dentin } & Silane-treated barium glass, Silica (Amorphous), UDMA, & Fusio \\
& TEGDMA, HEMA, 4-MET, Photo Curing System & \\
& Primer: acetone, HEMA, water, phosphoric monomer, & \\
BLB-CR6 & Bis-GMA, TEGDMA, others & LLB \\
& Besin Composite: CQ, Zirconium filler, TEGDMA, Bis-GMA, \\
& Primer: HEMA, MDP, water, photo-initiator, & \\
Clearfil MegaBond & Bonding: silanated colloidal silica, Bis-GMA, MDP, HEMA, & MB \\
(Clearfil SE Bond) & photo-initiator, others & MB+BF \\
BEAUTIFIL Flow F02 & Bis-GMA, TEGDMA, Glass filler & BF \\
\hline
\end{tabular}

UDMA: urethane dimethacrylate; TEGDMA: triethyleneglycol dimethacrylate; HEMA: 2-hydroxyethyl metacrylate; 4-MET: 4-methacryloxyethyl trimellitic acid; CQ: camphorquinone; Bis-GMA: bisphenol-A-diglycidyl methacrylate; Bis-MPEPP: 2,2'bis(4-methacryloxy polyethoxyphenyl) propane; MDP: 10-methacryloyloxydecyl phosphate 


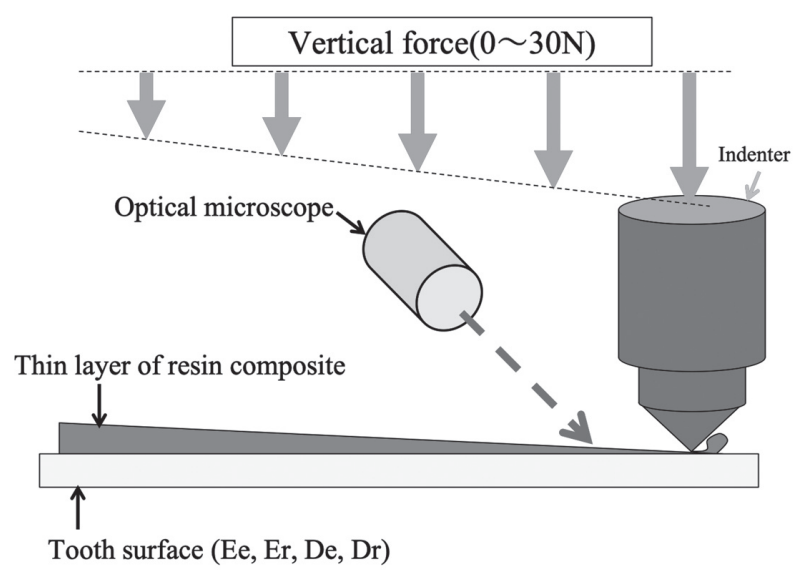

Fig. 1 Schematic drawing of scratch bond strength test.

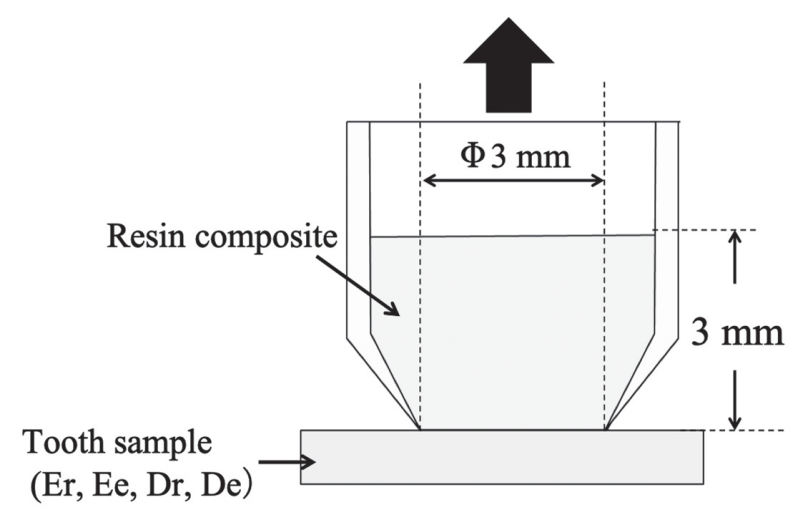

Fig. 2 Diagram of the specimens for testing tensile bond strength between tooth surface and coating materials.

Large arrow shows the direction of tensile force.

film bond strength), which was obtained from the scratch test, was evaluated by determining the critical load at which the coated material failed or the enamel or dentin surface began to appear.

\section{Tensile bond strength between tooth surface and coating materials}

A Teflon mold (inner diameter: $3 \mathrm{~mm}$, depth: $3 \mathrm{~mm}$ ) was placed on the surface of Ee, De, Er or Dr. The mold was then filled with Fusio, which was light-cured for $20 \mathrm{~s}$. The mold for LLB was filled with it; and, after primer application, the resin was light-cured in the same manner as for Fusio. For $\mathrm{MB}+\mathrm{BF}$, a primer for $\mathrm{MB}$ was applied to the surface of Ee, De, Er or Dr, followed by the bonding agent and curing. After that, the mold was filled with BF, which was then light-cured by the same procedure as used for the two other materials. Ten specimens of each material on each tooth surface were tested for tensile bond strength by using a universal testing machine (Autograph AGS-X, SHIMADZU, Kyoto, Japan) at a cross-head speed of $0.5 \mathrm{~mm} / \mathrm{min}$ (Fig. 2). The tensile bond strength, expressed in $\mathrm{MPa}$,

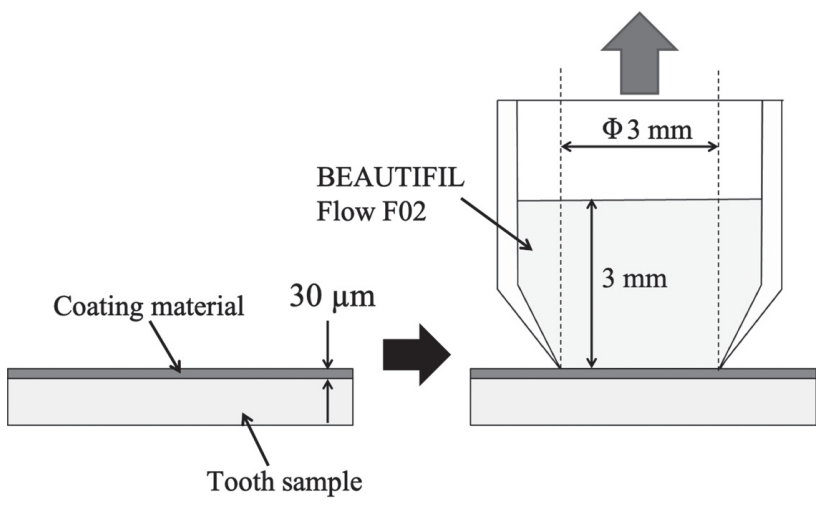

Fig. 3 Diagram of the specimens for tensile bond strength test between material coating tooth surface and resin composite.

Firstly, each material of about $30 \mu \mathrm{m}$ in thickness was coated on the tooth surface. Then, flowable resin composite was build up and tested with tensile force. Large arrow shows the direction of tensile force.

was calculated as the maximum load at failure divided by the cross-sectional area of the surface. After testing, fracture surfaces on the tooth side of the specimens were observed with a stereomicroscope.

Tensile bond strength between material coating tooth surface and resin composite

A plastic tape (approximately $30 \mu \mathrm{m}$ in thickness) was put on the outside of each treated tooth surface (Ee, De, Er, Dr) for regulating the thickness of the coating materials. Each material was applied to the tooth surface by procedures previously described to create a thin layer of material. A Teflon mold (same size as previously stated) was put on the coated surface; and then the mold was filled with a flowable resin composite (BF), which was light-cured for $20 \mathrm{~s}$. Ten specimens of each material on each tooth surface were tested for tensile bond strength by the same procedures as used for the previous testing (Fig. 3).

\section{Statistical analysis}

The obtained data were analyzed by one-way ANOVA and Sheffe's multiple comparison test $(\alpha=0.05)$.

\section{RESULTS}

SEM observation of eroded and remineralized tooth surfaces

Figure 4 presents SEM images of enamel (E) and dentin (D) surfaces before treatment, the eroded surfaces (Ee and De), and the remineralized (Er and Dr) ones. Grinding and polishing lines, as well as smear, were evident on both E and D surfaces. For the Ee group, the rough surface was due to exposed enamel rods; because the smear layer had been removed from the superficial surface. For the De group, the smear layer was removed, 
and opened dentin tubules were observed. For Er and Dr groups, crystal deposits were seen on part of the surface.

\section{Micro hardness}

Table 2 shows the results for Vicker's hardness number (VHN) for each tooth surface (Ee, De, E and D) and for the flowable resin composites. For the tooth surface, the difference between Ee and E, and that between De and $\mathrm{D}$, was statistically significant $(p<0.05)$. For the resin
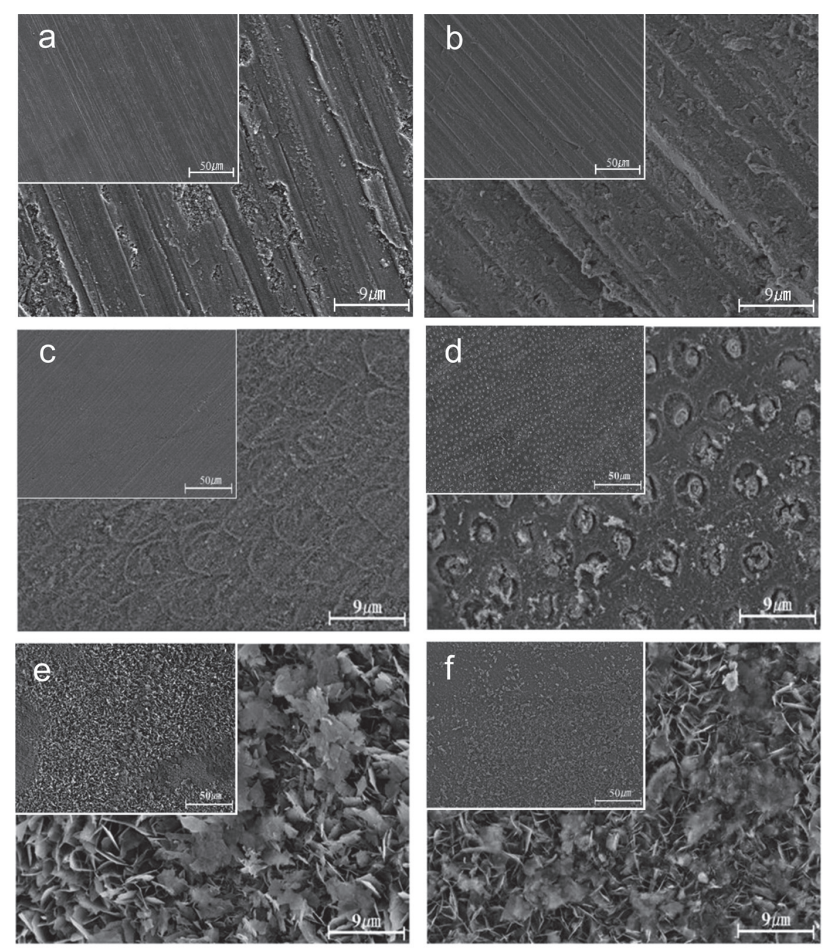

Fig. 4 SEM images of enamel and dentin surfaces before treatment, the eroded surfaces, and the remineralized ones.

The image on the upper left side of each photo is a lower-magnification image $(\times 500)$. The large image was obtained at high magnification $(\times 2,500)$. a: enamel before treatment (E), b: dentin before treatment (D), c: eroded enamel (Ee), d: eroded dentin (De), e: remineralized enamel (Er), f: remineralized dentin $(\mathrm{Dr})$. composites, Fusio had the highest value among all three materials $(p<0.05)$.

\section{Bond strength determined by the scratch test}

Figure 5 shows representative example of scratch and several parameters recorded for sliding damage. Figure 5 a shows several parameters affected by the test for bond strength between the tooth surface and thin-layer resin composite. The curves indicated abrupt changes in scratch depth (line 1), frictional coefficient (line 2), frictional force (line 3). Acoustic emission is shown by line 4. Figures 5(b, c, d) show optical microscopy images of scratched specimen at the points (A, B, and C) indicated in the figure. At point $\mathrm{A}$, the coating layer was scratching in progress (Fig. 5b). At point B, the coating material was peeled off completely (Fig. $5 \mathrm{c}$ ). At point $\mathrm{C}$, the coating material had been peeled off, and the tooth surface underneath was scratched (Fig. 5d). The critical load values for coating material-tooth surface failures were judged by considering the several parameters and the sliding damages.

Table 3 shows scratch strength of each material on the enamel (Ee and Er) and dentin (De and Dr) surfaces. For the enamel surface with Fusio, the value for Er was higher than that for Ee $(p<0.05)$. For LLB and MB+BF, there was no significant difference between $\mathrm{Er}$ and Ee samples $(p>0.05)$. In the case of the dentin surface, there was no significant difference between Er and Ee samples for any of the materials $(p>0.05)$. For the eroded-surface group (Ee and De), there was no statistically significant difference among materials ( $p>0.05)$.

Tensile bond strength test between tooth surface and coating materials

Table 4 shows the value of the tensile bond strength between treated enamel (Ee and Er) or dentin (De and Dr) surface and bulk (3-mm thickness) or thin-layer (30$\mu \mathrm{m}$ thickness) flowable resin composites. For the bulk sample groups, the bond strength for LLB on Ee was higher than that for it on Er. There was no significant difference between Er sample and Ee sample for bond strength with respect to Fusio or $\mathrm{MB}+\mathrm{BF} \quad(p>0.05)$. For dentin, LLB and MB+BF bond strengths on De were lower than those on Dr. There was no significant difference between De sample and Dr sample on Fusio $(p>0.05)$. For comparison among materials on eroded surfaces, $\mathrm{MB}+\mathrm{BF}$ showed higher bond strength than

Table 2 Vicker's hardness number for each tooth surface and resin composite (mean $\pm \mathrm{SD} ; n=5$ )

\begin{tabular}{cccc}
\hline Tooth surface & VHN & Resin composite & VHN \\
\hline E & $367.1 \pm 56.0^{\mathrm{a}}$ & Fusio & $32.8 \pm 0.6^{\mathrm{a}}$ \\
Ee & $274.3 \pm 30.7^{\mathrm{b}}$ & LLB & $26.7 \pm 0.7^{\mathrm{b}}$ \\
D & $75.4 \pm 6.7^{\mathrm{c}}$ & BF & $25.6 \pm 0.4^{\mathrm{b}}$ \\
De & $67.2 \pm 4.4^{\mathrm{d}}$ & - & - \\
\hline
\end{tabular}

The difference in letters in each column indicates that the values are statistically significant $(p<0.05)$. 


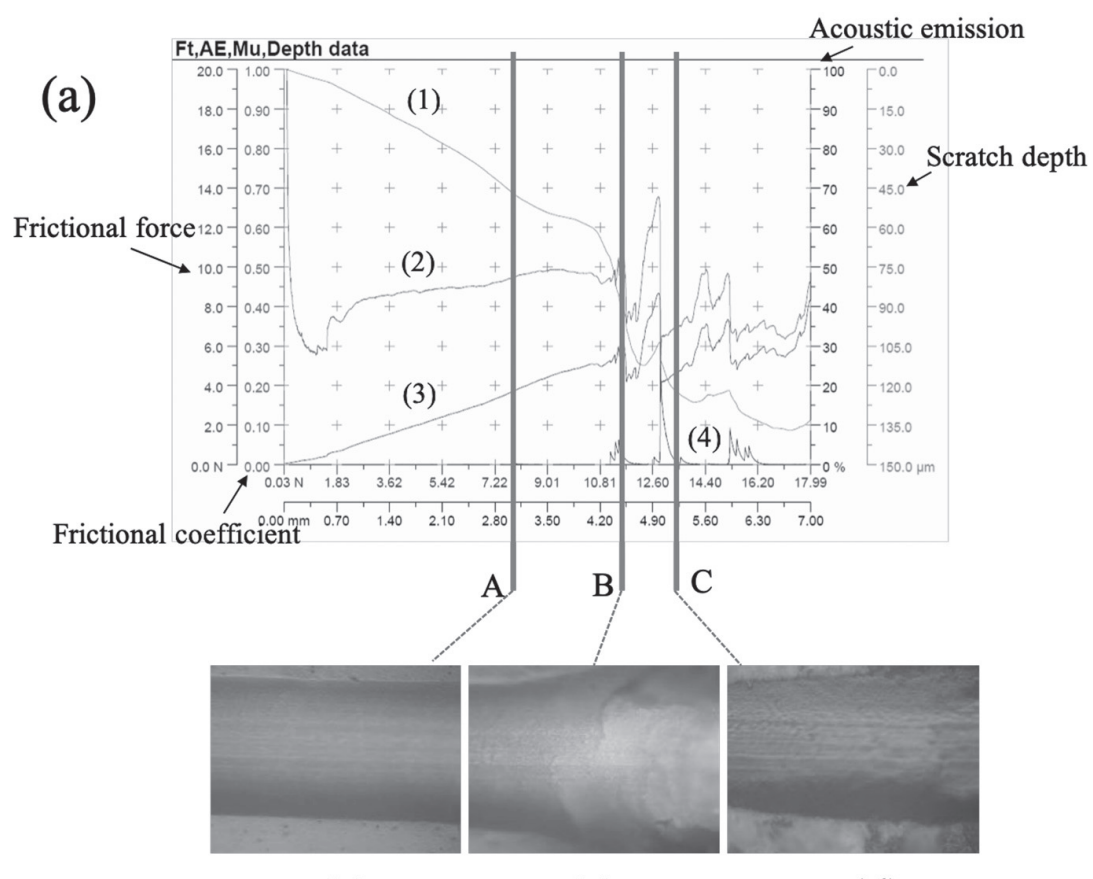

(b)

(c)

(d)

Fig. 5 Representative scratch test results and several parameters recorded for sliding damage as a result of the scratch test.

a: Graph of the results for several parameters. Lines (1), (2), (3), and (4) indicate scratch depth, frictional coefficient, frictional force, and acoustic emission, respectively. b, c, d: the microscopic image at points $\mathrm{A}, \mathrm{B}$, and $\mathrm{C}$ on the graph, respectively. At point $\mathrm{A}$, the coating material remained. At point B, the coating material was peeled off, and the tooth structure was exposed. At point C, the tooth structure was scratched.

Table 3 Bond strength $(\mathrm{N})$ assessed by scratch test between various tooth surfaces and each composite material (mean \pm SD; $n=10)$

\begin{tabular}{|c|c|c|c|c|}
\hline & \multicolumn{2}{|c|}{ Enamel } & \multicolumn{2}{|c|}{ Dentin } \\
\hline & Er & $\mathrm{Ee}$ & Dr & $\mathrm{De}$ \\
\hline Fusio & $24.6 \pm 4.3^{\mathrm{a}}$ & $18.9 \pm 5.9^{\mathrm{bc}}$ & $21.2 \pm 6.0^{\mathrm{a}}$ & $19.1 \pm 6.6^{\mathrm{a}}$ \\
\hline LLB & $19.7 \pm 3.4^{\mathrm{ab}}$ & $20.1 \pm 3.5^{\mathrm{ab}}$ & $21.2 \pm 2.5^{\mathrm{a}}$ & $22.5 \pm 3.0^{\mathrm{a}}$ \\
\hline $\mathrm{MB}+\mathrm{BF}$ & $13.8 \pm 2.2^{\mathrm{c}}$ & $17.8 \pm 2.2^{\mathrm{bc}}$ & $22.7 \pm 2.7^{\mathrm{a}}$ & $20.0 \pm 3.7^{\mathrm{a}}$ \\
\hline
\end{tabular}

The difference in letters indicates that the values are statistically significant for enamel or dentin $(p<0.05)$.

Table 4 Tensile bond strength (MPa) between various tooth surfaces and each composite material (mean $\pm \mathrm{SD} ; n=10)$

\begin{tabular}{|c|c|c|c|c|c|c|c|c|}
\hline & \multicolumn{4}{|c|}{ Enamel } & \multicolumn{4}{|c|}{ Dentin } \\
\hline & \multicolumn{2}{|c|}{ Bulk } & \multicolumn{2}{|c|}{ Thin } & \multicolumn{2}{|c|}{ Bulk } & \multicolumn{2}{|c|}{ Thin } \\
\hline & Er & $\mathrm{Ee}$ & Er & $\mathrm{Ee}$ & $\mathrm{Dr}$ & $\mathrm{De}$ & $\mathrm{Dr}$ & De \\
\hline Fusio & $7.8 \pm 1.8^{\mathrm{ab}}$ & $7.9 \pm 2.0^{\mathrm{ab}}$ & $6.8 \pm 2.2^{\mathrm{a}}$ & $9.2 \pm 3.5^{\mathrm{ab}}$ & $5.6 \pm 2.6^{\mathrm{ab}}$ & $2.9 \pm 1.2^{\mathrm{a}}$ & $1.9 \pm 1.9^{\mathrm{a}}$ & $1.4 \pm 1.2^{\mathrm{a}}$ \\
\hline LLB & $5.5 \pm 1.8^{\mathrm{a}}$ & $9.8 \pm 2.6^{\mathrm{b}}$ & $9.2 \pm 1.5^{\mathrm{ab}}$ & $13.7 \pm 3.8^{\mathrm{b}}$ & $10.1 \pm 3.4^{\mathrm{c}}$ & $5.8 \pm 1.1^{\mathrm{ab}}$ & $10.1 \pm 3.4^{\mathrm{bc}}$ & $9.4 \pm 2.1^{b}$ \\
\hline $\mathrm{MB}+\mathrm{BF}$ & $16.3 \pm 3.7^{\mathrm{c}}$ & $14.0 \pm 2.2^{\mathrm{c}}$ & $11.6 \pm 3.1^{\mathrm{b}}$ & $10.8 \pm 2.2^{\mathrm{ab}}$ & $14.8 \pm 3.3^{\mathrm{d}}$ & $9.1 \pm 2.4^{\mathrm{bc}}$ & $12.9 \pm 1.5^{\mathrm{cd}}$ & $15.5 \pm 2.1^{\mathrm{d}}$ \\
\hline
\end{tabular}

Bulk: 3-mm thickness of composite material, Thin: 30- $\mu \mathrm{m}$ thin layer of composite material.

The difference in letters indicates that the values are statistically significant for enamel or dentin and Bulk or Thin $(p<0.05)$. 
$\square$ Cohesive $\square$ Mix $\boxminus$ Interfacial

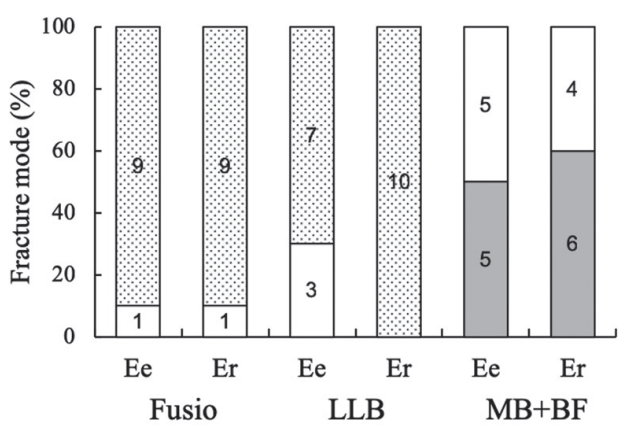

Enamel $\square$ Cohesive $\square$ Mix $\quad$ Interfacial

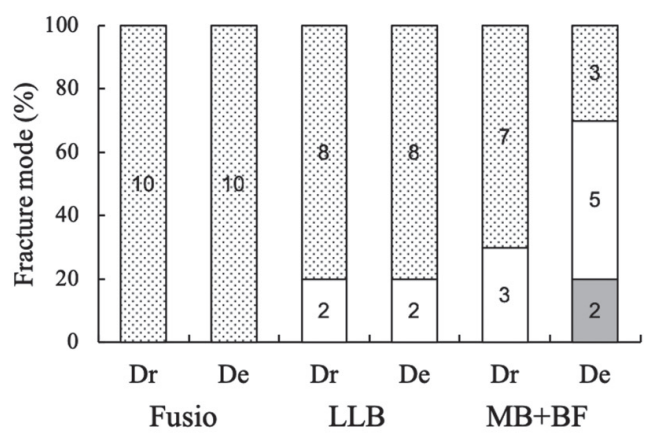

Dentin

Fig. 6 Fracture mode after tensile bond strength testing of the bulk-sample group. Dotted bars indicate interfacial fracture; white bars, mixed fracture; and gray bars, cohesive fracture at composite resin site.
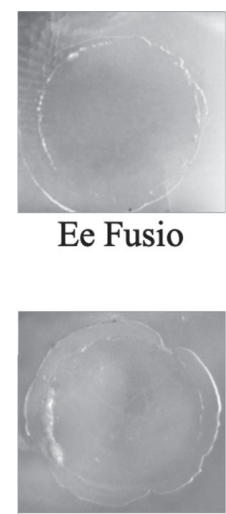

De Fusio

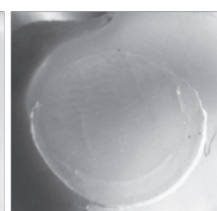

Er Fusio

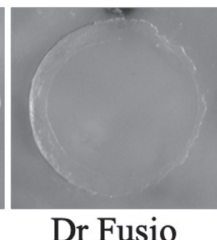

Dr Fusio

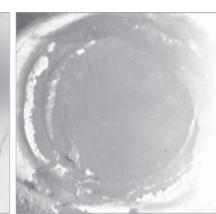

Ee LLB

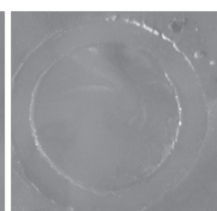

De LLB

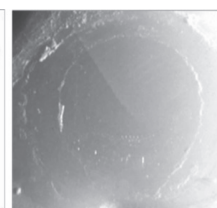

Er LLB

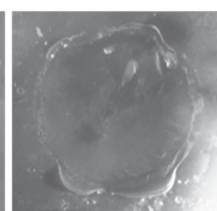

Dr LLB

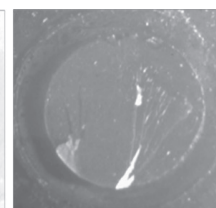

Ee

$\mathrm{MB}+\mathrm{BF}$

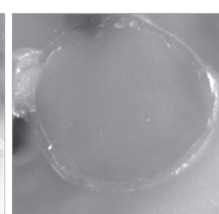

De $\mathrm{MB}+\mathrm{BF}$

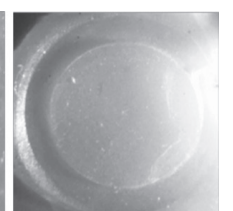

$\mathrm{Er}$ $\mathrm{MB}+\mathrm{BF}$

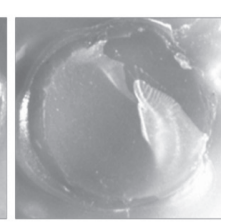

Dr

$\mathrm{MB}+\mathrm{BF}$

Fig. 7 Representative images of fracture surface after tensile strength testing of each composite in the bulk-sample group.

other materials on Ee; and Fusio, on De $(p<0.05)$. There was no significant difference between Fusio and LLB on either eroded surface $(p>0.05)$.

In the case of the thin-layer sample groups, when the eroded and remineralized surfaces were compared, there was no significant difference in bond strength between either enamel or dentin surface and any of the composite materials. When bulk and thin-layer data for the eroded surface were compared, the 30- $\mu$ m-layer specimens of Fusio and LLB tended to have higher bond strength toward Ee than the 3-mm thickness ones. For the eroded dentin (De), the bond strength of 30- $\mu \mathrm{m}$-layer specimens of LLB or MB+BF was higher than 3-mm thickness ones. However, Fusio showed lower for them.

Figures 6 and 7 show the fracture mode after testing and representative images of fracture surfaces, respectively, for the bulk sample groups. For the eroded samples (Ee and De), Fusio and LLB ones indicated fracture at the interface in almost all of the specimens.
LLB samples tended to have more mixed fractures than the Fusio ones. $\mathrm{MB}+\mathrm{BF}$ showed the same ratio of mixed fractures and cohesive fractures on Ee; on the other hand, there were many interface fractures on De. For remineralized samples (Er and Dr), almost all Fusio specimens indicated fracture at the interface with Er and Dr, as in the case of the eroded samples. LLB showed interface fractures on almost all specimens and mixed fractures on part of them. For MB+BF samples, the Er samples showed a ratio of mixed and cohesive fractures similar to that for the Ee samples. Dr samples indicated a higher rate of mixed fractures than that for De samples, and some Dr specimens also showed cohesive fractures.

Figures 8 and 9 indicate fracture mode after testing and show representative images of fracture surfaces, respectively, for the thin-layer sample groups. For Ee and De, Fusio showed the ratio of mixed fractures to be larger than that for the bulk samples. Almost all LLB 
$\square$ Cohesive $\square$ Mix $\square$ Interfacial

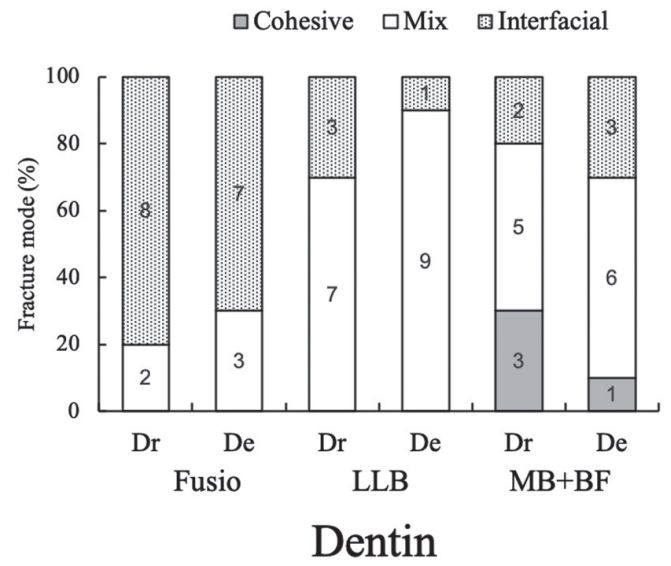

Fig. 8 Fracture mode after tensile strength testing of each composite in the thin-sample group. Dotted bar indicates interfacial fracture; white bar, mixed fracture; and gray bar, cohesive fracture at composite resin site.

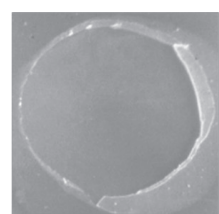

Ee Fusio

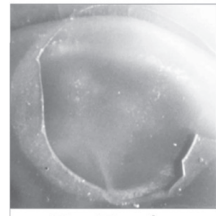

De Fusio

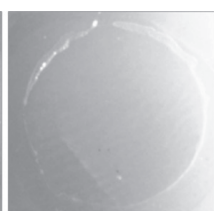

Er Fusio

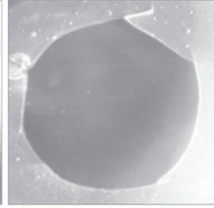

Dr Fusio

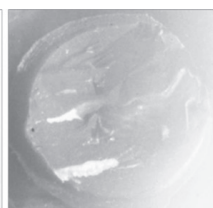

Ee LLB

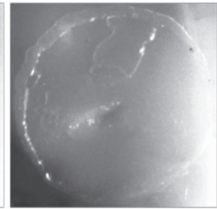

De LLB

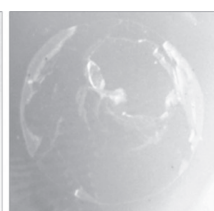

Er LLB

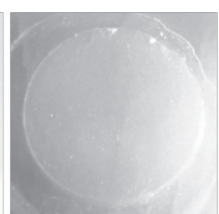

$\mathrm{Ee}$ $\mathrm{MB}+\mathrm{BF}$

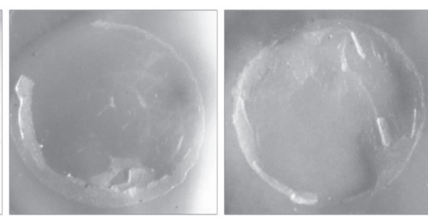

De

$\mathrm{MB}+\mathrm{BF}$

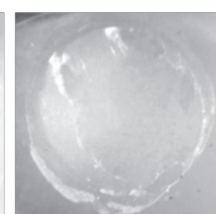

Er

$\mathrm{MB}+\mathrm{BF}$

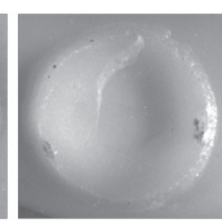

$\mathrm{Dr}$

$\mathrm{MB}+\mathrm{BF}$

Fig. 9 Representative images of fracture surface after tensile strength testing of each composite material in the thin-sample group.

specimens showed mixed fractures. Thin-layer MB+BF on Ee showed a higher ratio of cohesive fractures than that for the bulk samples. For Er and Dr samples, Fusio and LLB indicated a similar tendency of fracture mode, as did Ee and De samples. $\mathrm{MB}+\mathrm{BF}$ showed a ratio of cohesive fractures similar to that for the bulk samples.

\section{DISCUSSION}

Tooth erosion is one of the types of tooth wear, and it might accelerate the loss of resistance to such wear ${ }^{12}$. This present research was done with the ultimate goal of inhibiting tooth erosion. The artificially eroded teeth, which we created by immersion in a carbonated beverage, showed a rough enamel surface and open dentinal tubules in the dentin by SEM observation. Also, there was a statistically lower hardness value for the eroded teeth than for the non-demineralized teeth in this study. Earlier, Wongkhantee et al. ${ }^{13)}$ reported that a cycle of immersion in carbonated beverage (Cola) or artificial saliva leads to decreased hardness of the enamel or dentin surface. Other investigators studied erosion (demineralization) by using lactic $\operatorname{acid}^{14,15)}$, acetic acid ${ }^{16,17)}$, phosphoric $\operatorname{acid}^{18)}$, EDTA ${ }^{19,20)}$, or $\mathrm{pH}$ cycling ${ }^{21,22}$. However, these various solutions were used for creating artificial caries or decalcification, not for tooth wear. Acidic carbonated beverage (Cola) was used for producing erosion in this study, because it would simulate the acidic erosion commonly caused by this popular beverage.

Some self-adhesive resin materials are used for luting; however, there are few purposed as filling materials. So, there are no reports in the literature about bond strength between a self-adhesive filling material and eroded enamel or dentin.

In this study, the new scratch test was employed to evaluate bond strength between adhesives and treated enamel or dentin surfaces. Tensile and shear bond 
strength tests are the major ones used for evaluation of bond strength. However, these tests do not accurately reflect adhesive failure between the bonding agent and tooth surface, because these tests show various modes of failure ${ }^{10)}$. This new scratch test measured the force needed to delaminate a thin-film adhesive from the surface and expressed the bond strength between bonding agent and tooth surface. This scratch test is useful for assessing the bonding between a thin layer of material and a certain substrate. However, thick specimens (e.g., bonding between resin composite and tooth surface with an adhesive system) cannot be examined by using this test, because an indenter is required to apply a force to scratch between the specimens and other surface.

The results of bond-strength assessment by the new scratch test showed that only the Fusio sample indicated a lower strength in the erosion (Ee) group than in the remineralized (Er) group. Other composite samples on enamel or dentin did not show any decrease in bonding strength due to erosion. For determining bond strength by use of the scratch test, the required force for delamination from the base material (enamel or dentin) of thin-film composite specimens is taken as the bond strength ${ }^{10)}$. In the present study, the composite materials were used as a coating material on the tooth surface, and so we considered that the scratch test might be useful for our objective. The values obtained by this test may depend on the indentation hardness of materials ${ }^{10)}$. Our results seem to be related to the strength (micro hardness) of materials used. Fusio had a higher hardness value than the two other composite materials. The ratio or composition of filler included in the materials might have been different between Fusio and the others.

As for tensile bond strength, there was no significant difference between eroded and remineralized surface when thin and bulk samples of Fusio were examined. For comparison of the failure mode, the ratio of interfacial fractures for thin samples was lower than that for the bulk ones. In many groups, thin samples expressed higher tensile strength than bulk samples. In the case of bulk samples, when the composite materials were cured, shrinkage on curing and/or insufficient curing might have occurred. So tensile stress may have been applied at the interface between tooth and material, and/or incomplete adhesion might have occurred at this interface. As for thickness of adhesive, when adhesive thickness is increased, a significant increase is observed in stress concentration at the free edge and in the length of the stress-affected area $^{23}$. An excessive thickness of adhesive layer can negatively influence the strength and the quality of adhesion ${ }^{24)}$. The recommended usage for these materials is as a bonding material or tooth-coating material for demineralized (eroded) tooth surfaces.

The eroded enamel or dentin surface exhibited a lower bond strength than did sound enamel or dentin, probably due to the lower amount of minerals, higher porosity of the surface, and enlargement of the intercrystal spaces. These changes indicate that demineralized enamel is different from sound enamel, which may lead to an unsatisfactory etching pattern and infiltration of monomers, resulting in reduced bond strength ${ }^{21}$. Casas et al. ${ }^{25)}$ stated that immersion of a tooth in Cola decreases the bond strength between enamel and resin. On the other hand, acid-eroded enamel has a higher bond strength than that of normal enamel ${ }^{26}$. Erosion was not affected for the bond strength between enamel or dentin and resin ${ }^{27,28)}$.

The used adhesive monomer was 4-methacryloxyethyl trimetellitic acid (4-MET) for Fusio and phosphoric acid monomer for LLB. These monomers confer chemical bonding potential to hydroxyapatite and tooth tissue ${ }^{29)}$. Chemical bonding and diffusion into the enamel or dentin lead to good adhesive performance on demineralized enamel or dentin. On the other hand, the results of a tensile bond strength test showed Fusio to have a significantly lower bond strength than $\operatorname{LLB}^{7}$. Various sizes of bubbles with an average diameter of $2 \mu \mathrm{m}$ are observed between dentin and Fusio by TEM ${ }^{7}$; and these bubbles may be attributed to residual water droplets on the dentin surface and to the poor permeability of the initial flowable composite resin layer applied onto the dentin surface ${ }^{7}$. Both Fusio and LLB showed lower bond strength than MB as a two-step self-etching system. Self-adhesive resin composites might be an incomplete material at this present time.

\section{CONCLUSION}

The self-adhesive flowable resin composites indicated no significantly different bond strength between the eroded and remineralized tooth surface, though the conventional two-step adhesive showed a higher bond strength than the self-adhesive resin composite. This limited study suggests that these self-adhesive flowable resin composites might be useful as a tooth-coating material for acid-eroded tooth surfaces.

\section{ACKNOWLEDGMENTS}

This research was supported by a Grant-in Aid for Scientific Research from the Japan Society for the Promotion of Science (JSPS KAKENHI; Grant Number:JP25253100).

\section{REFERENCES}

1) Nunn J, Shaw L, Smith A. Tooth wear: dental erosion. Br Dent J 1996; 180: 349-352.

2) Green J. Prevention and management of tooth wear: the role of dental technology. Prim Dent J 2016; 5: 30-33.

3) Bartlett DW, Evans DF, Anggiansah A, Smith BG. A study of the association between gastro-oesophageal reflux and palatal dental erosion. Br Dent J 1996; 181: 125-131.

4) Meurman JH, ten Cate JM. Pathogenesis and modifying factors of dental erosion. Eur J Oral Sci 1996; 104: 199-206.

5) Attin T, Zirkel C, Hellwig E. Brushing abrasion of eroded dentin after application of sodium fluoride solutions. Caries Res 1998; 32: 344-350.

6) Sorvari R, Meurman JH, Alakuijala P, Frank RM. Effect of fluoride varnish and solution on enamel erosion in vitro. Caries Res 1994; 28: 227-232. 
7) Fu J, Kakuda S, Pan F, Hoshika S, Ting S, Fukuoka A, et al. Bonding performance of a newly developed step-less all-inone system on dentin. Dent Mater J 2013; 32: 203-211.

8) Tanuma T, Kotake H, Kusakabe S, Hotta M. Bond strength of self-adhesive flowable composite resin to human dentin. Jpn J Conserv Dent 2014; 57: 73-82.

9) Kusakabe S, Hotta M. Bond strength of one-step bonding agent to dentin measured by scratch test. Jpn J Conserv Dent 2013; 56: 298-309.

10) Kusakabe S, Rawls HR, Hotta M. Relationship between thin-film bond strength as measured by a scratch test, and indentation hardness for bonding agents. Dent Mater 2016; 32: e55-e62.

11) Sieck B, Takagi S, Chow LC. Assessment of loosely-bound and firmly-bound fluoride uptake by tooth enamel from topically applied fluoride treatments. J Dent Res 1990; 69: 1261-1265.

12) Johansson AK, Omar R, Carlsson GE, Johansson A. Dental erosion and its growing importance in clinical practice: from past to present. Int J Dent 2012; 2012: 1-17.

13) Wongkhantee S, Patanapiradej V, Maneenut C, Tantbirojn D. Effect of acidic food and drinks on surface hardness of enamel, dentine, and tooth-coloured filling materials. J Dent 2006; 34: 214-220.

14) Millar BJ, Abiden F, Nicholson JW. In vitro caries inhibition by polyacid-modified composite resins ('compomers'). J Dent 1998; 26: 133-136.

15) Zhou SL, Zhou J, Watanabe S, Watanabe K, Wen LY, Xuan $K$. In vitro study of the effects of fluoride-releasing dental materials on remineralization in an enamel erosion model. J Dent 2012; 40: 255-263.

16) Pereira PN, Inokoshi S, Tagami J. In vitro secondary caries inhibition around fluoride releasing materials. J Dent 1998; 26: 505-510

17) Okuyama K, Kadowaki Y, Matsuda Y, Hashimoto N, Oki S, Yamamoto $\mathrm{H}$, et al. Efficacy of a new filler-containing root coating material for dentin remineralization. Am J Dent 2016; 29: 213-218

18) Iijima M, Ito S, Nakagaki S, Kohda N, Muguruma T, Saito $\mathrm{T}$, et al. Effects of immersion in solution of an experimental toothpaste containing S-PRG filler on like-remineralizing ability of etched enamel. Dent Mater J 2014; 33: 430-436.

19) Sano H, Ciucchi B, Matthews WG, Pashley DH. Tensile properties of mineralized and demineralized human and bovine dentin. J Dent Res 1994; 73: 1205-1211.

20) Nakajima M, Okuda M, Pereira PNR, Tagami J, Pashley DH. Dimensional changes and ultimate tensile strengths of wet decalcified dentin applied with one-bottle adhesives. Dent Mater 2002; 18: 603-608.

21) Tedesco T, Soares FZM, Grande RHM, Rodrigues-Filho LE, Rocha RDO. Effect of cariogenic challenge on bond strength of adhesive systems to sound and demineralized primary and permanent enamel. J Adhes Dent 2014; 16: 1-8.

22) Okuyama K, Nakata T, Pereira PNR, Kawamoto C, Komatsu H, Sano H. Prevention of artificial caries: effect of bonding agent, resin composite and topical fluoride application. Oper Dent 2006; 31: 135-142.

23) Neves A de A, Coutinho E, Poitevin A, Van der Sloten J, Van Meerbeek B, Van Oosterwyck H. Influence of joint component mechanical properties and adhesive layer thickness on stress distribution in micro-tensile bond strength specimens. Dent Mater 2009; 25: 4-12.

24) D'Arcangelo C, Vanini L, Prosperi GD, Di Bussolo G, De Angelis F, D'Amario M, et al. The influence of adhesive thickness on the microtensile bond strength of three adhesive systems. J Adhes Dent 2009; 11: 109-115.

25) Casas-Apayco LC, Dreibi VM, Hipólito AC, Graeff MSZ, Rios $\mathrm{D}$, Magalhães AC, et al. Erosive cola-based drinks affect the bonding to enamel surface: an in vitro study. J Appl Oral Sci 2014; 22 : 434-441.

26) Lenzi T, Hesse D, Guglielmi C, Anacleto K, Raggio DP. Shear bond strength of two adhesive materials to eroded enamel. J Contemp Dent Pract 2013; 14: 700-703.

27) Navarro R, Vicente A, Ortiz AJ, Bravo LA. The effects of two soft drinks on bond strength, bracket microleakage, and adhesive remnant on intact and sealed enamel. Eur J Oral Sci 2011; 33: 60-65.

28) Cruz JB, Lenzi TL, Tedesco TK, Guglielmi CAB, Raggio DP. Eroded dentin does not jeopardize the bond strength of adhesive restorative materials. Braz Oral Res 2012; 26: 306312.

29) Yoshida Y, Nagakane K, Fukuda R, Nakayama Y, Okazaki M, Shintani $\mathrm{H}$, et al. Comparative study on adhesive performance of functional monomers. J Dent Res 2004; 83: 454-458. 